\title{
A Survey on Semantic Web Service Discovery Methods
}

\author{
Priyadharshini.G \\ PG Scholar \\ Sri Ramakrishna Engg Coll \\ Tamil Nadu, India
}

\author{
Gunasri.R \\ PG Scholar \\ Sri Ramakrishna Engg Coll \\ Tamil Nadu, India
}

\author{
Saravana Balaji.B \\ Assistant Professor \\ Sri Ramakrishna Engg Coll \\ Tamil Nadu, India
}

\begin{abstract}
Semantic web services focuses on providing automation and dynamics to existing web service technologies. A large amount of effort and money has been invested in the field of semantic web service discovery. In traditional web service discovery techniques, users need to select relevant web services manually from an extensive textual list. This paper provides an in-depth analysis on the existing approaches available for the discovery of semantic web services.
\end{abstract}

Keywords: Web service discovery, Semantics, UDDI, WSDL, ontology.

\section{INTRODUCTION}

Semantic Web Service (SWS) lies at the intersection of two significant trends in the evolution of the World Wide Web. The first is the rapid development of Web service technologies and the second is the advent of the Semantic Web. Existing web service protocols and languages need to be standardized for integrating the heterogeneous business solutions. Automated web service discovery still remains a complex task. This complexity is mainly due to the rapid increase in the number of web services available on the Web and the various concept models like Web Service Description Language (WSDL), Web Ontology Language for Services (OWL-S), Web Service Description Language Semantics (WSDL-S), Web Service Modelling Language (WSML) etc., with which the services are being exposed.

An important issue in the automation of web services is the discovery of web services using Universal Description Discovery and Integration (UDDI) [1]. The UDDI registry based discovery is insufficient for automatic service discovery because UDDI supports only keyword based matching. Querying in UDDI is based on category. To overcome these insufficiencies, Web Service Description Language (WSDL) based discovery techniques have emerged. Traditional syntactic approaches for dynamic service composition meet serious difficulties when multiple parties are involved [2].

With the advent of ontology and various semantic service matching algorithms [3], web services can be automatically discovered. There are three steps involved in the web service discovery process (see fig: 1). In the first step, developers advertise their web service. A web service description file written in WSDL, by the providers of the web service, is used to register web services in public repositories or service registry for advertisement [4]. The second step involves the request for the advertised web services by users. Users send request to web service repository by specifying the requirement in a pre-defined format. The hub part of the web service discovery model is the Web service matcher which matches user request with the available web services. The last step is selecting the exact web service from the retrieved web services.

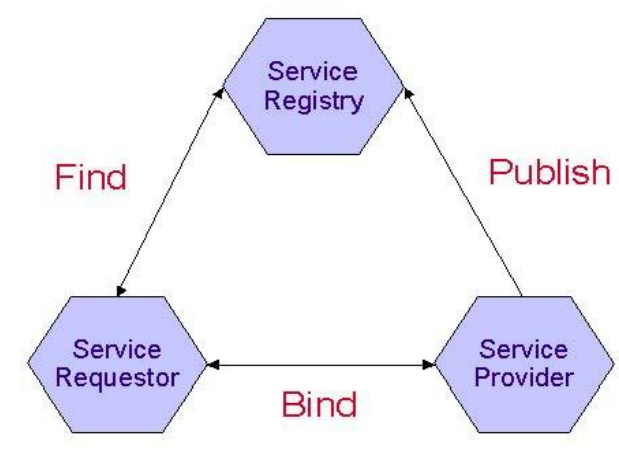

Fig: 1 Discovery of web services

Three factors that mainly affect the web service discovery process are:

1. Ability of the service providers to describe their services

2. Ability of the service requestors to describe their requirements

3. "Intelligence" of the service matchmaking algorithm.

In this paper, section 2 describes about some of the issues regarding semantic web service discovery process. Section 3 presents six web service discovery approaches like Context aware discovery, Publish Subscribe Model, Keyword Clustering, Layered Based Semantic Web Discovery, Service Advertisements in MANETs (SAM): Service Discovery in Heterogeneous Networks and Agent Based Discovery considering QoS parameter.

\section{ISSUES WITH TRADITIONAL SEMANTIC WEB SERVICE DISCOVERY SERVICES}

Web service discovery is defined as the process of matching user requests with the available web services. Web service can be identified by a service name and a service description written using standard WSDL. The standardization of the web service description makes the discovery process easier [5]. Several approaches have been proposed to improve the accuracy of Web service discovery by applying several techniques like data mining [7] [6], singular vector decomposition [8], graph based methods, ontology based discovery frameworks, agent based and logic based methods.

Information matching can be done at two levels:

a. In syntactic matching, the similarity of data is found using syntax driven techniques. The values between 0 and 1 are used to define the similarity amongst two concepts. 0 indicates that the concepts are completely 
dissimilar and 1 indicates that the concepts are completely similar.

b. In semantic matching, the key idea is the mapping of meanings between the concepts.

The techniques used for web service discovery should meet certain criteria. These criteria are:

- $\quad$ Functional Web Service Properties

What a web service can accomplish is described as the capability of the web service. These capabilities are described by its functional properties [9].

- Non-Functional Web Service Properties

The characteristics of a web service, i.e. how the web service can accomplish what it is capable of , is described by Non-functional properties in terms of QoS [9].

- An Effective Graphical User Interface

The ease with which the user can find and use the available web services is very crucial in Web Service Discovery process. This can be ensured by providing an effective graphical user interface (GUI).

- Classification Capability

While discovering the available web services, categorizing the web services will aid in the discovery process. For this reason, an effective classification method must be used to categorize the web services.

- Search or Browsing Facility

When a large number of web services are contained within a dataset, it is difficult for the user to find the appropriate service. To simplify this search process, search and browse facilities should be provided to the user. To further refine the search results of the user, filters can be provided.

- Ranking Function

Ranking the search results will help to identify the most relevant web services, for the user, within the search result list. Ranking will further enhance the usability of the web service discovery process.

- Result List Sort Capability

Users will have specific preferences while discovering the web services. The web service discovery mechanism should allow the user to sort the web service search result list in the order of the user's preference.

\section{SEMANTIC WEB SERVICE DISCOVERY METHODS}

\subsection{Context Aware Web Service discovery}

The format for sending a web service request is fixed. Some of the data in a user's request may be lost while transferring the request through the network. To overcome this problem, Context Aware Web Service discovery approach was suggested by Wenge Rong and Kecheng Liu [10]. Context aware discovery is useful for request optimization, result optimization and personalization. Context in web service discovery is formally defined as any information can explicitly and implicitly affect the user's web service request generation.

Context is divided into two categories - explicit and implicit. Explicit context is directly provided by the user during the matchmaking process like Q\&A information. Implicit context is collected in either automatic or semi-automatic manner. Implicit context is more applicable to web service discovery as the user is not directly involved. Context awareness is divided into four categories depending on how context is collected. The categories are personal profile oriented context, usage history oriented context, process oriented context and other context. Under circumstances in which a single web service is not sufficient to complete the user request, composition of multiple web services can be carried out. In this case, context should be built considering composite web service discovery process. This approach is better than the traditional keyword matching.

\subsection{Publish Subscribe Model}

A Publish Subscriber model for web service discovery was proposed by Falak Nawz, Kamram Quadir and H.Farooq Ahmad [11]. Service notification priority is provided to service requestors to discover the available services. Semantic based web service matching is used here. Service descriptions are matched using OWL-S and concept matching. Web services are ranked depending on the scores assigned.

The system is divided into two phases - subscription phase and notification phase. In the subscription phase, a subscriber registers onto a registry for notification of the required services. In the notification phase, a new service is published on the registry. When a user subscribes, the subscription information along with the user's location and web service requirements are stored in subscription knowledge base. This information stored in the knowledge base in OWL format can be used later for service matching.

The best matching web service is selected by matching user requirements to OWL-S descriptions stored in the registry. Matching can be done in one of the six levels as Exact, PlugIn, Subsume, Enclosure, Unknown and Fail. In the notification phase, OWLS description for newly registered web services are added to the matching subscription categories. The time required for web service discovery is minimized with this approach as the search area is reduced to the specified category. The probability of finding the most suitable web service also increases. The limitation of this approach is that it adds overhead by developing and maintaining new components in system architecture.

\subsection{Keyword clustering}

This approach based on Keyword clustering and concept expansion was suggested by J. Zhou, T. Zhang, H.Meng, L. Xiao, G. Chen and D. Li. This approach calculates similarity matrix of words based on Pareto principal. Matching service is determined using semantic reasoning and Bipartite graphs.

\subsection{Layer based semantic web service discovery}

Finding a matching web service in the entire service repository is a time consuming process.

Guo Wen-yue, Qu Hai-cheng and Chen Hong [12] have suggested a Layer based approach for semantic web service discovery. In this approach, search is divided into three layers by applying filters at each layer. Applying filters reduces the search area. The three layers for service matching are service category matching, service functionality matching and quality of service matching.

Semantic web service discovery is done based on OWL-S. ServiceProfile documents are used for service matching. The first step in the service discovery process is service category matching. The category of service is stored in the 
ServiceProfile. This value is matched against service category of the user request. If there is match the web service is selected to enter the next service layer.

Service functionality matching degree is calculated in the service functionality matching layer. The attributes - hasInput, hasOutput, hasPrecondition and hasResult are matched against the user service request. These attributes are defined in the ServiceProfile.

The third step is computing the quality of the service (QoS) matching degree. QoS is decided based on the response time and reliability of the service discovery system. From the above three steps service matching degree is calculated and the advertisements that best suit the requestors are presented in the form of a list.

\subsection{Service advertisements in MANETs (SAM): service discovery in heterogeneous networks}

Traditional mechanisms for web service discovery such as UDDI and ebXML are not suitable for heterogeneous and decentralized networks. A method was proposed by $\mathrm{F}$. Johnsen, T. Hafsoe, A. Eggen, C. Griwodz and P. Halvorsen [13] for web services discovery in such networks. Different networks use different protocols. Service discovery gateways are used so that the different networks can continue to use different protocols. Interoperability is ensured by using service discovery gateways that can translate between different service discovery mechanisms. This mechanism is called as Service Advertisements in MANETs (SAM).

A gateway periodically queries all services in the WSDirectory. Available services are then looked up in the gateway's local service cache. If a service is deleted from a domain, it is removed from the local cache.

\subsection{Agent based discovery considering QoS}

The best web services available can be selected by using QoS parameters. T. Rajendran and P. Balasubramanie [14] suggest a web service discovery method based on QoS parameters. These parameters are response time, availability, throughput and time. The proposed method contains an agent for ranking the various web services available based on the QoS certificates received from service publishers. Two main entities in the proposed method is verifier and certifier

The service publisher component is accountable for the registration, updation and deletion of web service information in UDDI. Service publisher is supplied by the service providers with QoS values related to business and performance of web services. Verification and certification of these QoS values is then done by web service discovery agent. The service functionality is then published to the UDDI registry.

The service consumer searches the UDDI registry for a service through the discovery agent. This agent aids in finding the best service available based on the QoS parameters. Time required for choosing the best web service with respect to the QoS values eventually decreases. QoS verification is the process of validating the information described in the service interface. The certification process makes use of this verification result.

Table 1 gives an overview of the various web service discovery approaches discussed above along with their advantages and disadvantages.

Table: 1 Methods of Web Service Discovery

\begin{tabular}{|c|c|c|c|}
\hline $\begin{array}{l}\text { S. } \\
\mathrm{N}\end{array}$ & $\begin{array}{c}\text { Web Service } \\
\text { Discovery } \\
\text { Method }\end{array}$ & Advantages & Disadvantages \\
\hline 1 & $\begin{array}{c}\text { Context aware } \\
\text { method }\end{array}$ & $\begin{array}{c}\text { This } \\
\text { approach is } \\
\text { useful for } \\
\text { request and } \\
\text { result } \\
\text { optimization } \\
\text { and } \\
\text { personalizatio } \\
\text { n. }\end{array}$ & $\begin{array}{l}\text { The complexity } \\
\text { of the system } \\
\text { architecture } \\
\text { increases when } \\
\text { new attributes and } \\
\text { constraints are } \\
\text { added. }\end{array}$ \\
\hline 2 & $\begin{array}{l}\text { Publish } \\
\text { subscribe } \\
\text { model }\end{array}$ & $\begin{array}{l}\text { The time } \\
\text { required for } \\
\text { web service } \\
\text { discovery is } \\
\text { minimized } \\
\text { because of } \\
\text { ranking of } \\
\text { web services. }\end{array}$ & $\begin{array}{l}\text { Publish and } \\
\text { subscribe systems } \\
\text { are tightly } \\
\text { coupled and } \\
\text { hence it is } \\
\text { difficult to } \\
\text { develop and } \\
\text { maintain these } \\
\text { systems. }\end{array}$ \\
\hline 3 & $\begin{array}{l}\text { Keyword } \\
\text { clustering }\end{array}$ & $\begin{array}{c}\text { Kuhn- } \\
\text { munkres } \\
\text { algorithm is } \\
\text { used here. } \\
\text { This } \\
\text { algorithm } \\
\text { computes the } \\
\text { optimal } \\
\text { match for the } \\
\text { user requests. }\end{array}$ & $\begin{array}{l}\text { Clustering is } \\
\text { based on } \\
\text { keywords and not } \\
\text { on the semantic } \\
\text { meaning. }\end{array}$ \\
\hline 4 & $\begin{array}{l}\text { Layer based } \\
\text { semantic web } \\
\text { service } \\
\text { discovery }\end{array}$ & $\begin{array}{l}\text { Since filters } \\
\text { are applied at } \\
\text { each layer, } \\
\text { the search } \\
\text { area is vastly } \\
\text { reduced. }\end{array}$ & $\begin{array}{l}\text { There is } \\
\text { complexity } \\
\text { involved in } \\
\text { calculating the } \\
\text { matching degree } \\
\text { at each layer. }\end{array}$ \\
\hline 5 & $\begin{array}{c}\text { SAM: Service } \\
\text { discovery in } \\
\text { heterogeneous } \\
\text { networks }\end{array}$ & $\begin{array}{c}\text { Supports } \\
\text { Web service } \\
\text { discovery in } \\
\text { heterogeneou } \\
\text { s and } \\
\text { decentralized } \\
\text { networks }\end{array}$ & $\begin{array}{l}\text { Synchronization } \\
\text { between the } \\
\text { services available } \\
\text { in the domain and } \\
\text { the local cache is } \\
\text { a difficult task. }\end{array}$ \\
\hline 6 & $\begin{array}{l}\text { Agent based } \\
\text { discovery } \\
\text { considering } \\
\text { QoS }\end{array}$ & $\begin{array}{c}\text { Best service } \\
\text { is discovered } \\
\text { by using the } \\
\text { QoS } \\
\text { parameters }\end{array}$ & $\begin{array}{c}\text { Other QoS } \\
\text { parameters are } \\
\text { not considered. }\end{array}$ \\
\hline
\end{tabular}

\section{CONCLUSION}

Traditional web service discovery mechanisms involve manual selection of required web services, by users, from an extensive textual list. This process is time-consuming and exhaustive. This paper has highlighted some of the approaches available for SWS discovery. Different web service discovery approaches and their advantages and disadvantages have been discussed. Most of the approaches differ in the way the web service matching is carried out. Some of the approaches are 
considering the concept of semantic web while some others focus on different information retrieval methods. Context aware discovery is useful for web service request optimization, result optimization and personalization of web service discovery. Publish Subscribe Model approach ranks the web services based on the service request priority. Keyword Clustering calculates a similarity matrix of words and finds the matching services by using semantic reasoning. Layered Based Semantic Web Discovery approach divides the search into three layers by applying filters to discover the accurate service. Service Advertisements in MANETs (SAM) uses gateways for interoperability among heterogeneous networks. Agent Based Discovery considers QoS parameters to discover the best service among available services.

Semantic web service discovery approaches reduces cost and time and enhances precision and recall by moving from manual services to reusable functionality. As traditional web service discovery mechanisms are time-consuming, solutions for automatic discovery are drawing more attention.

\section{REFERENCES}

[1] UDDI. org., UDDI Spec Technical Committee Draft,http://www.uddi.org/pubs/uddi_v3.htm\#_Toc85907 967

[2] Qingcong Lv, Jiliang Zhou, Qiying Cao, "Service Matching Mechanisms in Pervasive Computing Environments", International Workshop on Intelligent Systems and Applications, Wuhan, May 2009, pp. 1-4

[3] Umesh Bellur, Harin Vadodaria and Amit Gupta, "Semantic Matchmaking Algorithms", Advances in Greedy Algorithms, Source: Advances in Greedy Algorithms, Book edited by: Witold Bednorz, ISBN 978953-7619-27-5, I-Tech, Vienna, Austria, November 2008, pp. 586

[4] Christensen, E., Curbera, F., Meredith, G., Weerawarana, S.: Web Services Description Language (WSDL) 1.1, W3C Note (2001)

[5] XMethods.http://www.xmethods.com/
[6] Nayak R.: Using Data Mining In Web Services Planning, Development and Maintenance. International Journal of Web services Research, vol. 5, pp. 62-80 (2008)

[7] Nayak R., Lee B.: Web Service Discovery with additional Semantics and Clustering. In: IEEE/WIC/ACM International Conference on Web Intelligence (WI'07), Silicon Valley, USA (2007)

[8] Sajjanhar, A., Hou, J., Zhang, Y.: Algorithm for Web Services Matching. In: Proc. APWeb 2004, LNCS vol. 3007, pp 665-670, Springer Verlag, (2004)

[9] Stollberg, M. and M. Kerrigan, Goal-based Visualization and Browsing for Semantic Web Services, in Proceedings of Web Information Systems Engineering - WISE 2007 Workshops. 2007: Nancy, France.

[10] Rong, W., Liu, K.: A Survey of Context Aware Web Service Discovery: From User's Perspective. In: Fifth IEEE International Symposium on Service Oriented System Engineering (2010)

[11] 4. Nawaz, F., Qadir, K., Farooq Ahmad, H.: SEMREGPro: A Semantic based Registry for Proactive Web Service Discovery using Publish Subscribe Model. In Fourth International Conference on Semantics, Knowledge and Grid. IEEE Xplore (2008)

[12] Guo, W.-Y., Qu, H.-C., Chen, H.: Semantic web service discovery algorithm and its application on the intelligent automotive manufacturing system. In: International Conference on Information Management and Engineering. IEEEXplore (2010)

[13] Johnsen, F., Hafsøe, T., Eggen, A., Griwodz, C., Halvorsen, P.: Web Services Discovery across Heterogeneous Military Networks. IEEE Communications Magazine (October 2010)

[14] Rajendran, T., Balasubramanie, P.: An Optimal AgentBased Architecture for Dynamic Web Service Discovery with QoS. In: Second International Conference on Computing, Communication and Networking Technologies. IEEEXplore(2010). 